\title{
DChIPRep, an R/Bioconductor package for differential enrichment analysis in chromatin studies
}

Christophe D Chabbert, Lars M Steinmetz, Bernd Klaus

The genome-wide study of epigenetic states requires the integrative analysis of histone modification ChIP-seq data. Here, we introduce an easy-to-use analytic framework to compare profiles of enrichment in histone modifications around classes of genomic elements, e.g. transcription start sites (TSS). Our framework is available via the user-friendly R/Bioconductor package DChIPRep. DChIPRep uses biological replicate information as well as chromatin Input data to allow for a rigorous assessment of differential enrichment. DChIPRep is available for download through the Bioconductor project at http://bioconductor.org/packages/DChIPRep. Contact DChIPRep@gmail.com 


\title{
DChIPRep, an R/Bioconductor package for differential enrichment analysis in chromatin studies
}

\author{
Christophe D. Chabbert ${ }^{1}$, Lars M. Steinmetz ${ }^{2}$, and Bernd Klaus ${ }^{3}$ \\ ${ }^{1}$ Genome Biology Unit, European Molecular Biology Laboratory, 69117 Heidelberg, \\ Germany \\ Oncology iMed, CRUK-Cambridge Institute, AstraZeneca, Li Ka Shing Centre, Robinson \\ Way, Cambridge CB2 ORE, United Kingdom. \\ ${ }^{2}$ Genome Biology Unit, European Molecular Biology Laboratory, 69117 Heidelberg, \\ Germany, \\ Stanford Genome Technology Center, Stanford University, Palo Alto, CA 94304, USA \\ and \\ Department of Genetics, Stanford University School of Medicine, Stanford, CA 94305, \\ USA \\ ${ }^{3}$ Genome Biology Unit, European Molecular Biology Laboratory, 69117 Heidelberg, \\ Germany, corresponding author, Email: bernd.klaus@embl.de
}

\section{ABSTRACT}

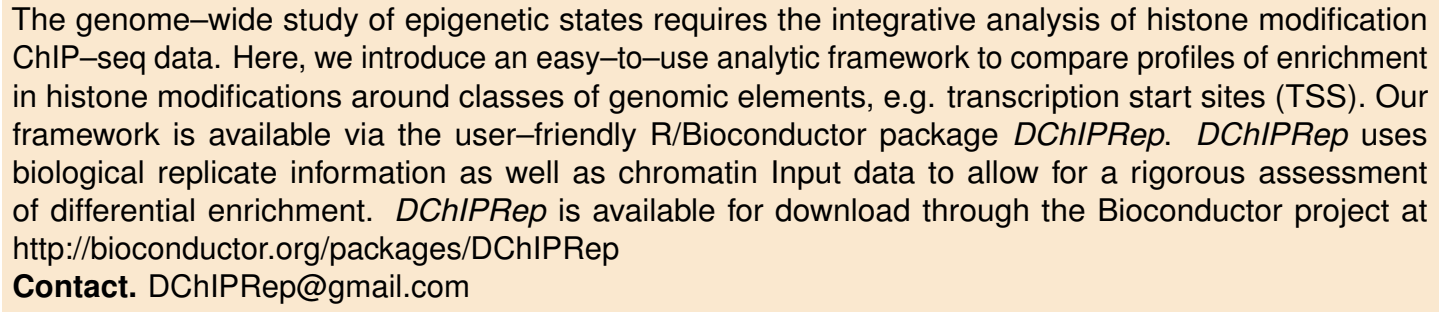
ChIP-seq data. Here, we introduce an easy-to-use analytic framework to compare profiles of enrichment in histone modifications around classes of genomic elements, e.g. transcription start sites (TSS). Our framework is available via the user-friendly R/Bioconductor package DChIPRep. DChIPRep uses biological replicate information as well as chromatin Input data to allow for a rigorous assessment of differential enrichment. DChIPRep is available for download through the Bioconductor project at http://bioconductor.org/packages/DChIPRep

Contact. DChIPRep@gmail.com

Keywords: Bioinformatics, Computational Biology, Genomics, ChiP-Seq, Chromatin, Histone-Modifications

\section{INTRODUCTION}

The elementary component of eukaryotic chromatin, the nucleosome, is composed of $147 \mathrm{bp}$ DNA fragments wrapped around an octamer comprising two copies of 4 of the histone proteins. The N-terminal tails of these proteins are subject to multiple post-translational modifications (PTM) including acetylation, phosphorylation and methylation (Lawrence et al., 2016). Such modifications may be found in combination on several residues of the same histone proteins, adding to the complexity of the combinatorial space of PTM that may be explored (Tan et al., 2011). Recent studies have highlighted the importance of these PTM in key cellular processes such as transcription, DNA replication and repair. Protocols based on chromatin immunoprecipitations followed by deep sequencing (ChIP-seq) allow for a genome-wide mapping of these modifications. Such endeavors have resulted in the generation of complex sequencing datasets that require appropriate bioinformatics tools to be analyzed. From this data, profiles of enrichment in histone modifications around classes of genomic elements, e.g. transcription start sites (TSS) are routinely computed. Once these enrichment profiles have been obtained, a common analysis task is to compare them between experimental conditions. However, due to a lack of tools tailored to the assessment of differential enrichment, these comparisons are often performed in a purely descriptive manner (e.g. by comparing plots of enrichment profiles around transcription start sites). In this article, we present a workflow to assess differential 
enrichment in a statistically rigorous way. This workflow is implemented in a user-friendly package named DChIPRep that is available via the Bioconductor project (Huber et al., 2015).

\section{Review of existing tools and approaches}

Several software tools designed to analyze certain aspects of histone modification data are already available. These usually focus on one or several of the 3 main aspects explored in chromatin biology: the genome-wide determination of nucleosome positions (not adressed by DChIPRep), the identification of genomic loci enriched in the modifications of interest (so-called peaks, not addressed by DChIPRep) and differential binding analysis, an aspect tackled by our package. Diverse statistical and numerical approaches have been concurrently implemented to infer nucleosome positions, including Fourier transform (nucleR, Flores and Orozco, 2011), Gaussian filtering (Genetrack, Albert et al., 2008), wavelets (NUCwave, Quintales et al., 2014) as well as probabilistic or Bayesian approaches (NucleoFinder Becker et al., 2013, PING 2.0 Woo et al., 2013, NOrMAL Polishko et al., 2012). Mutiple approaches based on signal smoothing and local background modeling have also been implemented to identify regions with high numbers of mapped reads (peaks) of variable width (Feng et al., 2012; Bailey et al., 2013).

Some algorithms proposed recently go beyond the determination of nucleosome or peak positions and aim at assessing differential enrichment. However, they commonly rely on the identification of regions of interest (e.g. around called peaks) using the ChIP-seq datasets themselves e.g. DiffBind, (Ross-Innes et al., 2012; Stark and Brown, 2011). Notably, csaw (Lun and Smyth, 2014) allows for a genome wide identification of differential binding events without an a priori specification of regions of interest. It uses a windowing approach and implements strategies for a post hoc aggregation of significant windows into regions. Although DESeq 2 is commonly used for differential binding analysis of ChIP-Seq data (Bailey et al., 2013), to the best of our knowledge, no direct approach to compare enrichment profiles of histone modifications around classes of genomic elements exists so far. Furthermore, most existing tools do not offer the possibility to directly correct for biases using the Input chromatin samples. Commonly, these profiles are analyzed in a purely descriptive manner and conclusions are drawn solely from plots of metagene/metafeature (e.g. transcription start site plots).

Here we present DChIPRep, an R/Bioconductor package designed to compute and compare histone modification enrichment profiles from ChIP-seq datasets at nucleotide resolution. The workflow implemented in DChIPRep uses both the biological replicate and the chromatin Input information to assess differential enrichment. By adapting an approach for the differential analysis of sequencing count data (Love et al., 2014), DChIPRep tests for differential enrichment at each nucleotide position of a metagene/metafeature profile and determines positions with significant differences in enrichment between experimental groups. An overview of the complete workflow is given next.

\section{Overview of the implemented framework}

The framework implemented in DChIPRep consists of three main steps:

1. The chromatin Input data is used for positionwise-normalization.

2. The methodology of Love et al. (2014) is used to perform positionwise testing. A minimum absolute $\log _{2}-$ fold-change greater than zero between the experimental groups is set during the testing procedure to ensure that called positions show an non-spurious differential enrichment.

3. Finally, in order to assess statistical significance, local False Discovery Rates (local FDRs, Strimmer, 2008) are computed from the p-values obtained as a result of the testing step. Local FDRs assess the significance of each positions individually and are thus well suited for the detection of fine-grained differences.

\section{Real data analysis}

We first apply DChIPRep and a modified version of its framework using methodology inspired by the csaw and edgeR (Lun and Smyth, 2014; McCarthy et al., 2012) packages to yeast ChIPseq data and compare the enrichment profiles around TSS in wild-type and mutant strains, 


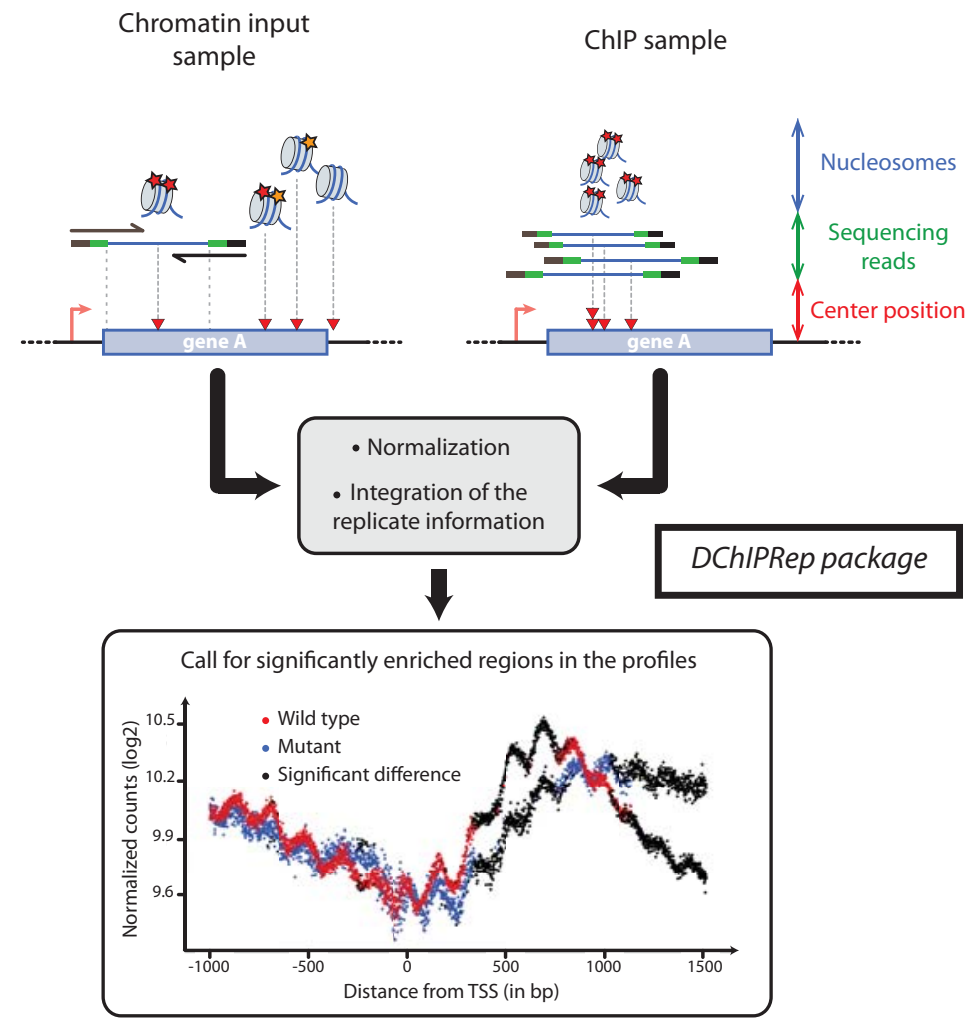

Figure 1. Illustration of the DChIPRep workflow. Chromatin Input- and ChIP-data are analyzed jointly and positions showing significantly different enrichment are identified using the replicate information.

demonstrating how our package can derive biological insights from large-scale sequencing datasets.

We furthermore analyze a published mouse data set by Galonska et al. (2015), to compare H3K4me3 enrichment around selected TSS in embryonic stem cells grown in two conditions (serum/LIF and 2i conditions).

\section{METHODS}

\section{General architecture of the package}

DChIPRep uses a single class DChIPRepResults that wraps the input count data and stores all of the intermediate computations. The testing and plotting functions are then implemented as methods of the DChIPRepResults object. The plotting functions return ggplot2 (Wickham, 2009) objects than can subsequently be modified by the end-user.

DChIPRep's analytical method uses histone modification ChIP-Seq profiles at single nucleotide resolution around a specific class of genomic elements (e.g. annotated TSS). In the case of paired-end reads originating from chromatin fragmented using microccocal nuclease (MNAse), such profiles can be obtained using the middle position of the genomic interval delimited by the DNA fragments (Fig. 1).

Thus, the variables characterizing the samples are the genomic positions relative to a specific class of genomic elements (e.g. TSS). These variables take the values given by the number of sequenced fragments with their center at these specific positions. The data is summarized across genomic features (e.g. genes or transcripts) at each of these nucleotide positions, so that metagene/metafeature profiles are obtained. The input data for DChIPRep can be alignment files in the SAM format or already processed count data. 
Data import

DChIPRep has two possible data input formats. The input data can be two count tables per sample (for ChIP and Input), with the genomic features used (e.g. genes or transcripts) in the rows and the position wise counts per genomic feature in the columns. Alternatively, one can provide two count tables for ChIP and Input that contain the data at the metafeature level, such that the data is summarized across individual genomic features. These tables then have one row per position relative to the genomic element (e.g. TSS) studied and one column per sample.

DChIPRep can either import tab-separated txt files (two files per experimental sample with data at the level of the individual genomic features) or two R count matrices for ChIP and Input data, which contain the data already summarized at a metafeature level (summarized across features per position). A table containing the experimental conditions and other sample specific annotation is needed as well. A Python script (DChIPRep.py) is also provided along with the package to generate suitable tab-separated input files from SAM alignment files of paired-end data and a gff annotation. The script relies on the HTSeq module (Anders et al., 2015) and may be customized via multiple parameters.

It also possible to directly import .bam files using the function importData_soGGi. The function also supports the import of single-end data and uses functionality from the soGGi package (Dharmalingam and Carroll, 2015). The resulting imported data is then a matrix of metafeature profiles.

Further details on the data import can be found in the package vignette, which is available via Bioconductor and as Supplemental_Item_1_vignette_DChIPRep.html.

\section{Computation of the metafeature profile}

Once the data is summarized on the feature level (i.e. count tables), we can compute the metafeature profiles with the function summarizeCountsPerPosition for each of the ChIPSeq and chromatin Input samples.

The function first filters out features with very low counts. Then, in order to summarize the data across features, a trimmed mean of the counts at each position is computed.

Finally, these positionwise mean values are multiplied by the number of features retained at each position. This way, a raw metafeature profile for each individual sample is obtained.

\section{Call for enriched regions}

The statistical approach implemented in DESeq2 is used to call for significantly deferentially enriched positions (Love et al., 2014).

Here, the chromatin input is used to compute normalization factors that correct for potential local biases in chromatin solubility, enzyme accessibility or PCR amplification.

Essentially, we use the positionwise ratios of ChIP and Input data as the normalized data: we first adjust the total counts (representing the library size) of the chromatin Input metafeature profiles so that they match the library size of their corresponding ChIP samples. This corrects for global differences in sequencing depth between the ChIP and Input samples and is commonly performed as a simple scaling normalization in the analysis of high-throughput sequencing data as exemplified in edgeR. The adjusted Input data is now on the same scale as the ChIP data and can directly be used as a position-specific normalization factor in DESeq2. Other reports have also shown that it is possible to use the Input data directly as an "ordinary" sample and then test for differences between ratios of ratios (Love, 2014) - this functionality might be added as an option in a future version of the package.

After specifying a minimum fold change, Wald tests are performed to assess significant changes in the metagene/metafeature profiles.

Finally, local FDRs estimated by the fdrtool (Strimmer, 2008) package are used to assess statistical significance based on the p-values obtained from the Wald-test.

All of these steps are implemented in the runTesting function (Fig. 1).

\section{Plotting functions}

DChIPRep provides two plotting functions to represent and inspect the final results of the analysis. The plotProfiles function summarizes the biological replicates by taking a robust 
position wise mean (Hampel et al., 2011) and then plots a smoothed enrichment profile around the genomic element class of interest (e.g. TSS).

The plotSignificance function plots the unsmoothed enrichment profile and highlights positions with a significant difference in enrichment as returned by the runTesting function (Fig. 1). The plotting functions return ggplot2 objects that can be easily customized.

While these plotting capabilities are relatively elementary, we would like to point out that the Bioconductor package soGGi as well as the standalone tool ngs.plot (Shen et al., 2014) offer more sophisticated visualizations of ChIP-seq profiles, which are not at the core of DChIPRep functionality.

\section{RESULTS}

\section{A yeast case study}

We applied DChIPRep to a paired-end ChIP-seq dataset for which biological replicates are available (Chabbert et al., 2015). This dataset also includes sequences from the associated chromatin inputs, which were obtained using MNAse digestion.For this particular case study, we have only selected the sequencing data generated using a classical ChIP-seq protocol (while other samples have been profiled using a different protocol in the same publication). Using the annotation from Xu et al., 2009, we compared the enrichment of the H3K4me2 mark in annotated ORFs (5170 items) in the wild type strain of Saccharomyces cerevisiae and the set $2 \Delta$ mutant. We have called a significant enrichment (local FDR $<0.2)$ in the mutant for 906 positions located within 1500bp downstream of the transcription start site (Fig. 1).

\section{Steps for a typical analysis}

In order to illustrate the usage of DChIPRep we document the series of simple commands that are needed to be to run a typical analysis.

After the data has been preproccesed, we first need to import a table that contains the annotation information for our samples. This table contains information on the count table file names and the desired number of up- and downstream positions to be compared, as well as the experimental group a sample belongs to. As mentioned above, details on the required format of the annotation table can be found in the package vignette in the supplement (Supplemental_Item_1_vignette_DChIPRep.html).

We can then import the data using the function importData.

$$
\begin{aligned}
& \text { Listing 1. Data Import } \\
& \text { sampleTable_K4me2 }<- \text { read.csv ("sampleTable_K4me2.csv") } \\
& \text { importedData }<- \text { importData (sampleTable_K4me2) }
\end{aligned}
$$

After then data import, we can perform the positionwise testing with the runTesting function, extract the results using the resultsDChIPRep function and finally obtain the significance plot in Fig. 1 via a call to the plot Significance function.

Listing 2. Results and Firgure

testResults $<-$ runTesting (importedData)

testResults $<-$ resultsDChIPRep(testResults)

plotSignificance (testResults)

\section{A comparison to an csaw/edgeR-based pipeline}

The framework implemented in DChIPRep uses the DESeq2-package (Love et al., 2014) to perform the statistical testing. The csaw-package (Lun and Smyth, 2014) implements a strategy based on methods implemented in edgeR (McCarthy et al., 2012) to assess differential binding in ChIP-Seq data sets genome-wide. While csaw and DChIPRep are not directly comparable, we can adapt the csaw framework to assess the differential enrichment (for a summary of the csaw framework, see section 1.3. of the csaw user guide at Bioconductor). 

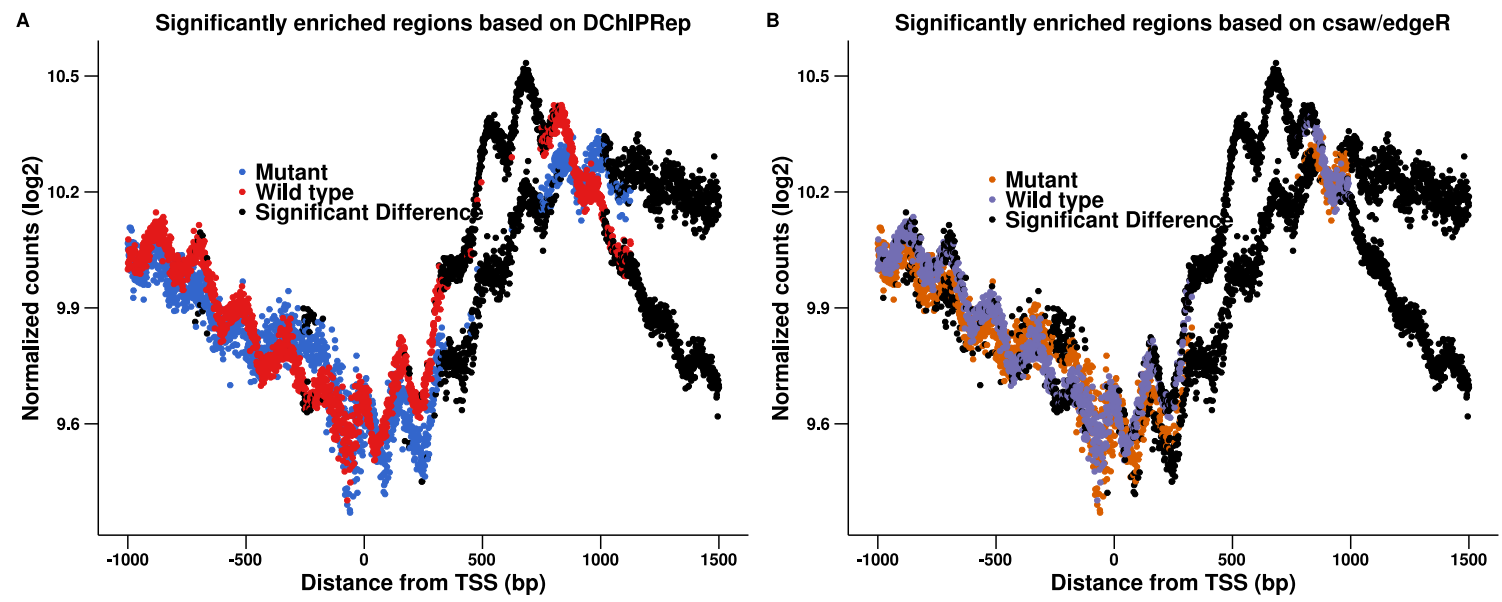

Figure 2. Results of the csaw/edgeR-based calling for enriched regions are shown in panel (B). The results presented in Figure 1 are shown again in Panel (A). We applied an edgeR-based testing to the data (instead of using DESeq2). This included a post hoc thresholding of the fold-changes. The figure shows that this pipeline calls many positions with moderate fold-changes up to 250p downstream of the TSS as significant.

Specifically, we used the log-normalization factors computed from the chromatin-input as offsets for the GLM-model and then applied the quasi-likelihood (QL) methods of Lund et al. (2012) to perform a dispersion shrinkage and an appropriate F-test to assess the differential enrichment. Note that since edgeR does not allow for an a priori specification of a fold change threshold, we had to specify it post hoc. The complete analysis can be found in supplementary file 2 - ReproduceFiguresDChIPRepPaper.zip.

Figure 2 shows the results of this approach. The modified pipeline identified 1171 positions as significantly deferentially enriched located within $1500 \mathrm{bp}$ downstream of the transcription start site. Comparing Fig. 2 to Fig. 1, we see that the $e d g e R$-based pipeline gives very similar results in this case. In fact, all 906 positions called by DESeq2 downstream of the TSS are also called by edgeR. This indicates that the local FDR based thresholding, which assesses the significance of the position individually is more important than the actual statistical test performed. We still observe that DChIPRep identifies deferentially enriched regions more consistently, while the edgeR pipeline calls many positions with moderate fold-changes up to 250p downstream of the TSS as significant. This might be due to the fact that a post hoc fold change thresholding had to be performed. DChIPRep would therefore be less prone to calling false positive as it is less sensible to weak enrichment (which might be resulting from intrinsic variability in the performance of immunoprecipitation for example).

\section{Analysis of $\mathrm{H} 3 \mathrm{~K} 4 \mathrm{me} 3$ profiles in mouse embryonic stem cells (ESCs)}

As an additional case study we analyzed the H3K4me3 enrichment profiles of mouse ESCs from Galonska et al. (2015). The data consists of two replicates in two conditions that correspond to a typical stem cell culture conditions (serum/LIF) and a $24 \mathrm{~h}-2 \mathrm{i}$ condition characterized by two inhibitors (2i) of the MEK and GSK3 pathways respectively (thought to represent an embryonically restricted ground state). As only one chromatin Input was sequenced (whole cell extract, WCE), we use this data as Input data for all four samples.

We downloaded the data from the short read archive (SRA) at the European Nucleotide Archive (ENA, accession PRJNA242892) and the lists of called peaks in two conditions from GEO (GSE56312).

The raw single-end reads were aligned to the $\mathrm{mm} 9$ reference genome using bowtie2 (Langmead and Salzberg, 2012) with default options. Then, filtering of unmapped, low mapping quality $(<10)$, duplicated and multi-mapping reads was performed using Picard tools (BroadInstitute, 2016). We then inferred the fragment length for each sample using using cross 


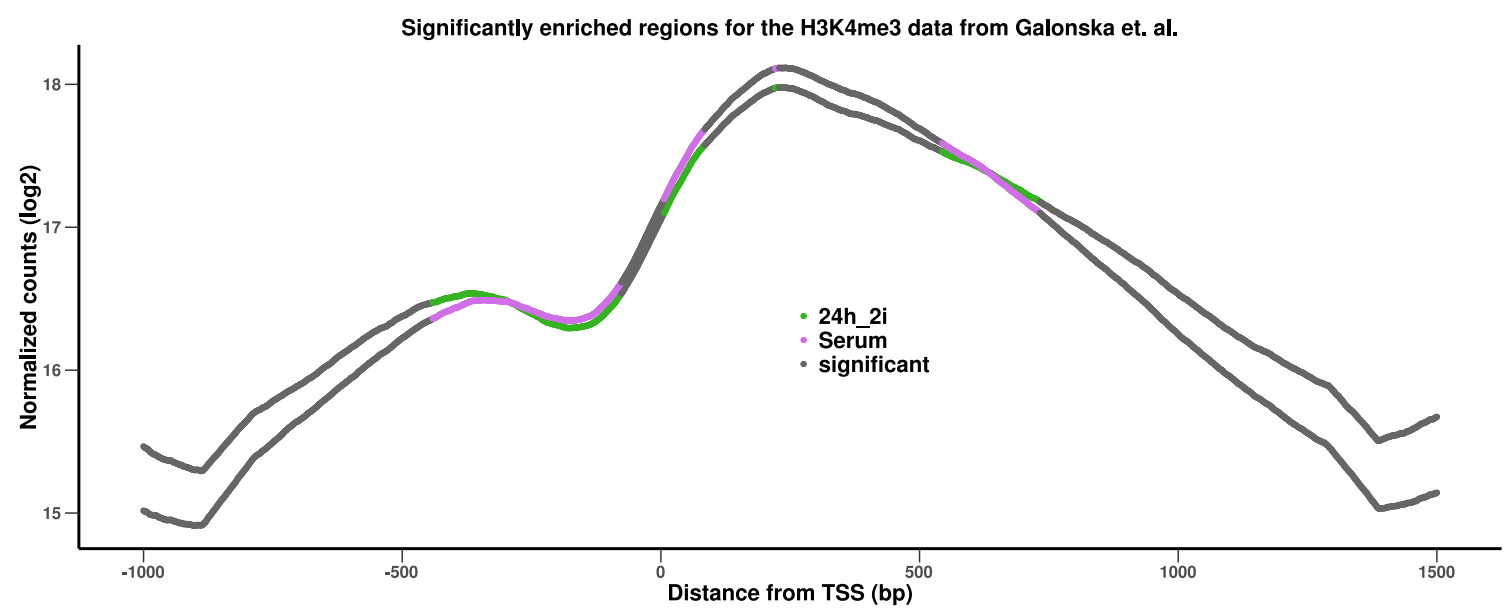

Figure 3. Results of the analysis of the H3Kme3 data from Galonska et al. (2015). The profiles are based on TSS close to identified peaks of the histone modification in the two conditions.

correlation plots from SPP (Kharchenko et al., 2008). We subsequently merged the two peak lists provided by the authors (GEO GSE56312) and then annotated the peaks to the closest mm9 TSS using the function annotatePeakInBatch from the package ChIPpeakAnno (Zhu et al., 2010; Zhu, 2013). We finally used the function regionPlot from the soGGi package (Dharmalingam and Carroll, 2015) to create metagene profiles around this subset of TSS that are close to the peaks identified by the authors of the original study. We kindly refer the reader to the the preprocessing-code in the supplemental information for additional details (Supplemental_Item_3_PreprocessingGalonskaData.html).

Figure 3 shows that the profiles in both conditions are quite smooth and very similar. We therefore used DChIPRep with a log fold change threshold of 0 and a local FDR threshold of 0.3 to identify significantly different positions. The serum condition has a somewhat higher enrichment near the TSS, while the $24 \mathrm{~h}-2 \mathrm{i}$ condition is slightly more enriched upstream and downstream of the TSS. The genomic regions where both profiles become very similar in shape and intensity are correctly identified as not significantly differentially enriched. While the overall differences are subtle, DChIPRep successfully and correctly identifies regions with differences, provided suitable parameters are used.

\section{Reproducible research}

The complete code and the data used for the case study can be found in the supplementary material (Supplemental_Item_2_ReproduceFiguresDChIPRepPaper.zip).

\section{DISCUSSION AND CONCLUSION}

The package DChIPRep provides an integrated analytical framework for the computation and comparison of enrichment profiles from replicated ChIP-seq datasets at nucleotide resolution or lower.

Starting from the primary alignment of paired-end reads, the software allows a rapid identification of significantly differentially enriched positions relative to classes of genomic elements and provides straightforward plotting of the enrichment profiles.

We also applied the DChIPRep-package to two published data sets using yeast and mouse a model systems. The yeast case study demonstrates DChIPRep's favourable performance when compared to a pipline inspired by the csaw-package for differential binding analysis.

\section{Acknowledgements}

We thank Sophie Adjalley, Vicent Pelechano, Aleksandra Pekowska and Alejandro Reyes for helpful discussions and critical comments on the manuscript. 


\section{REFERENCES}

Albert, I., Wachi, S., Jiang, C., and Pugh, B. F. (2008). GeneTrack-a genomic data processing and visualization framework. Bioinformatics, 24(10):1305-1306.

Anders, S., Pyl, P. T., and Huber, W. (2015). Htseq-a python framework to work with highthroughput sequencing data. Bioinformatics (Oxford, England), 31(2):166-169.

Bailey, T., Krajewski, P., Ladunga, I., Lefebvre, C., Li, Q., Liu, T., Madrigal, P., Taslim, C., and Zhang, J. (2013). Practical guidelines for the comprehensive analysis of chip-seq data. PLoS computational biology, 9(11):e1003326.

Becker, J., Yau, C., Hancock, J. M., and Holmes, C. C. (2013). NucleoFinder: a statistical approach for the detection of nucleosome positions. Bioinformatics, 29(6):711-716.

Broad-Institute (2016). Picard tools - by broad institute.

Chabbert, C. D., Adjalley, S. H., Klaus, B., Fritsch, E. S., Gupta, I., Pelechano, V., and Steinmetz, L. M. (2015). A high-throughput ChIP-seq for large-scale chromatin studies. Molecular Systems Biology, 11(1):777-777.

Dharmalingam, G. and Carroll, T. (2015). soggi: Visualise chip-seq, mnase-seq and motif occurrence as aggregate plots summarised over grouped genomic intervals. R package version 1.3.2.

Feng, J., Liu, T., Qin, B., Zhang, Y., and Liu, X. S. (2012). Identifying chip-seq enrichment using macs. Nature protocols, 7(9):1728-1740.

Flores, O. and Orozco, M. (2011). nucleR: a package for non-parametric nucleosome positioning. Bioinformatics, 27(15):2149-2150.

Galonska, C., Ziller, M. J., Karnik, R., and Meissner, A. (2015). Ground state conditions induce rapid reorganization of core pluripotency factor binding before global epigenetic reprogramming. Cell Stem Cell, 17(4):462-470.

Hampel, F., Hennig, C., and Ronchetti, E. (2011). A smoothing principle for the huber and other location m-estimators. Computational Statistics \& Data Analysis, 55(1):324-337.

Huber, W., Carey, J., V., Gentleman, R., Anders, S., Carlson, M., Carvalho, S., B., Bravo, C., H., Davis, S., Gatto, L., Girke, T., Gottardo, R., Hahne, F., Hansen, D., K., Irizarry, A., R., Lawrence, M., Love, I., M., MacDonald, J., Obenchain, V., Ole's, K., A., Pag'es, H., Reyes, A., Shannon, P., Smyth, K., G., Tenenbaum, D., Waldron, L., Morgan, and M. (2015). Orchestrating high-throughput genomic analysis with Bioconductor. Nature Methods, 12(2):115-121.

Kharchenko, P. V., Tolstorukov, M. Y., and Park, P. J. (2008). Design and analysis of ChIP-seq experiments for DNA-binding proteins. Nat Biotechnol, 26(12):1351-1359.

Langmead, B. and Salzberg, S. L. (2012). Fast gapped-read alignment with bowtie 2. Nature Methods, 9(4):357-359.

Lawrence, M., Daujat, S., and Schneider, R. (2016). Lateral thinking: How histone modifications regulate gene expression. Trends in genetics : TIG, 32(1):42-56.

Love, M. I. (2014). Deseq2 testing ratio of ratios (rip-seq, clip-seq https://support.bioconductor.org/p/61509/).

Love, M. I., Huber, W., and Anders, S. (2014). Moderated estimation of fold change and dispersion for RNA-seq data with DESeq2. Genome Biol, 15(12):550.

Lun, A. T. L. and Smyth, G. K. (2014). De novo detection of differentially bound regions for ChIP-seq data using peaks and windows: controlling error rates correctly. Nucleic Acids Research, 42(11):e95-e95.

Lund, S. P., Nettleton, D., McCarthy, D. J., and Smyth, G. K. (2012). Detecting differential expression in RNA-sequence data using quasi-likelihood with shrunken dispersion estimates. Statistical Applications in Genetics and Molecular Biology, 11(5).

McCarthy, D. J., Chen, Y., and Smyth, G. K. (2012). Differential expression analysis of multifactor RNA-seq experiments with respect to biological variation. Nucleic Acids Research, 40(10):42884297.

Polishko, A., Ponts, N., Roch, K. G. L., and Lonardi, S. (2012). NORMAL: accurate nucleosome positioning using a modified gaussian mixture model. Bioinformatics, 28(12):i242-i249.

Quintales, L., Vázquez, E., and Antequera, F. (2014). Comparative analysis of methods for genome-wide nucleosome cartography. Brief Bioinform, 16(4):576-587.

Ross-Innes, C. S., Stark, R., Teschendorff, A. E., Holmes, K. A., Ali, H. R., Dunning, M. J., Brown, 
G. D., Gojis, O., Ellis, I. O., Green, A. R., Ali, S., Chin, S.-F., Palmieri, C., Caldas, C., and Carroll, J. S. (2012). Differential oestrogen receptor binding is associated with clinical outcome in breast cancer. Nature.

Shen, L., Shao, N., Liu, X., and Nestler, E. (2014). ngs.plot: Quick mining and visualization of next-generation sequencing data by integrating genomic databases. BMC genomics, 15:284.

Stark, R. and Brown, G. (2011). Diffbind: differential binding analysis of chip-seq peak data. Bioconductor - http://bioconductor.org/packages/release/bioc/html/DiffBind.html.

Strimmer, K. (2008). A unified approach to false discovery rate estimation. BMC Bioinformatics, 9(1):303.

Tan, M., Luo, H., Lee, S., Jin, F., Yang, J. S., Montellier, E., Buchou, T., Cheng, Z., Rousseaux, S., Rajagopal, N., Lu, Z., Ye, Z., Zhu, Q., Wysocka, J., Ye, Y., Khochbin, S., Ren, B., and Zhao, Y. (2011). Identification of 67 histone marks and histone lysine crotonylation as a new type of histone modification. Cell, 146(6):1016-1028.

Wickham, H. (2009). ggplot2: Elegant Graphics for Data Analysis. Springer-Verlag New York.

Woo, S., Zhang, X., Sauteraud, R., Robert, F., and Gottardo, R. (2013). PING 2.0: an r/bioconductor package for nucleosome positioning using next-generation sequencing data. Bioinformatics, 29(16):2049-2050.

Xu, Z., Wei, W., Gagneur, J., Perocchi, F., Clauder-Münster, S., Camblong, J., Guffanti, E., Stutz, F., Huber, W., and Steinmetz, L. M. (2009). Bidirectional promoters generate pervasive transcription in yeast. Nature, 457(7232):1033-1037.

Zhu, L. J. (2013). Integrative analysis of chip-chip and chip-seq dataset. Methods in molecular biology (Clifton, N.J.), 1067:105-124.

Zhu, L. J., Gazin, C., Lawson, N. D., Pagès, H., Lin, S. M., Lapointe, D. S., and Green, M. R. (2010). Chippeakanno: a bioconductor package to annotate chip-seq and chip-chip data. BMC bioinformatics, 11:237. 